\title{
Fast and Convenient Method For FE-SEM Characterization of Microstructured Organic Solutions in Ionic Liquids
}

\author{
Alexey S. Kashin and Valentine P. Ananikov
}

N.D. Zelinsky Institute of Organic Chemistry, Department of Structural Studies, Moscow, Russia.

Direct visualization of the morphology and dynamics of liquid phase systems by the means of microscopy is of great demand for life sciences, material science, chemistry and catalysis. Electron microscopy techniques allow to visualize the processes of interest in real time with high spatial resolution. Amongst all liquid media, ionic liquids (ILs) attract much attention due to wide applicability in energy production and storage, crude materials treatment, waste processing, organic synthesis and biochemistry. An important advantage of ILs is that they are suitable for direct electron microscopy measurements owing to their compatibility with electron microscope conditions including high vacuum in the specimen chamber.

In our studies we focused on the microscopy analysis of organic solutions based on ionic liquids. These challenging samples required specific observation conditions due to their high sensitivity to the electron beam irradiation and due to the absence of specific contrast in SEM. The simple and convenient method was proposed for the FE-SEM observations of liquid samples based on ionic liquids (1-butyl-3-methylimidazolium, 1-ethyl-3-methylimidazolium and 1-butylpyridinium ionic liquids). The samples of interest were applied on the standard copper TEM grids and studied directly in the secondary electron mode at low accelerating voltages. The dynamic behavior of the observed structures was recorded on SEM-video. Principle scheme of the analysis is given on the Figure 1.

With the use of developed technique a number of valuable results were obtained. The formation of micro-phase was detected in the mixtures of ionic liquids with water [1]. It was found that the structures of various shapes and sizes can be formed depending on the nature of the ionic liquid and on the water concentration. The diversity of morphologies from small micro-droplets for the trace amounts of water to two-dimensional channel-like structures for high water content was observed in the IL-water solutions. The possibility of employment of such media for toxic wastes storage and mechanism of the harmful compounds encapsulation at the micro-scale were established with the use of electron microscopy [2]. The proposed method was successfully applied to the series of hazardous substances: DDT, malathion (insecticides), glyphosate (herbicide), amoxicillin (antibiotic) and others. In the series of subsequent experiments the mechanism of dissolved compound extraction from solution in ionic liquids was revealed. The dependence of the extraction rate on the solution micro-structure for the model compound - dialanine (peptide) was observed directly by FESEM [3]. The proposed method of the SEM observation of organic solutions in ionic liquids was further extended to the multi-component reaction mixtures. Negative role of water in the acidcatalyzed conversion of fructose to an important platform compound 5-HMF was associated with micro-phase aggregation and visualized directly [1]. The examples of the obtained FE-SEM images are summarized on the Figure 2.

As a result of the study, the simple method for the observation of micro-structures in solutions and reaction mixtures based on ionic liquids has been developed. SEM analysis of ionic liquids performance in the storage and recovery of various compounds as well as in the biomass processing was successfully carried out without the use of any expensive and laborious sample preparation protocols [4]. 


\section{References:}

[1] AS Kashin et al, Angew Chem Int Ed 55 (2016), p. 2161.

[2] MM Seitkalieva et al, ACS Sustainable Chem Eng 6 (2018), p. 719.

[3] MM Seitkalieva et al, Sep Purif Technol 196 (2018), p. 318.

[4] This work was supported by the Russian Science Foundation (RSF grant 14-50-00126).

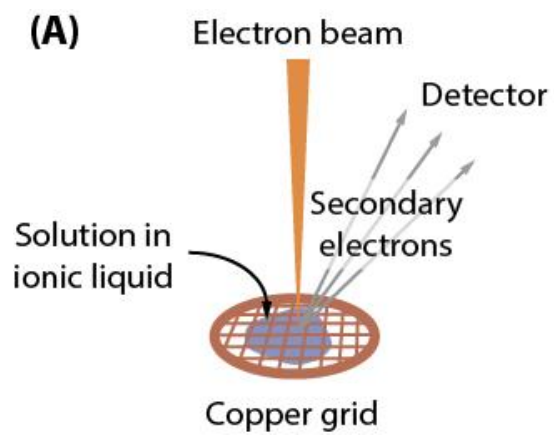

(B)

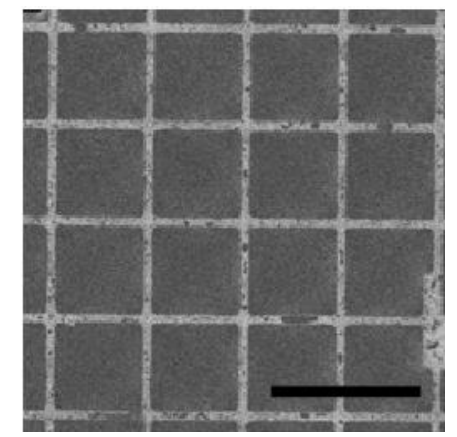

Figure 1. Developed technique for the direct FE-SEM analysis of mixtures based on ionic liquids: principal scheme of the analysis (A) and electron microscopy image of the copper grid filled with ionic liquid (B). Scale bar $-200 \mu \mathrm{m}$.

(A)

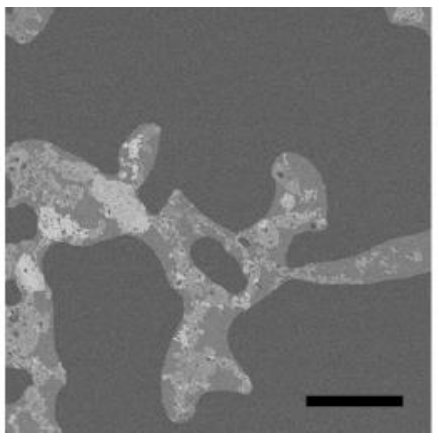

(B)

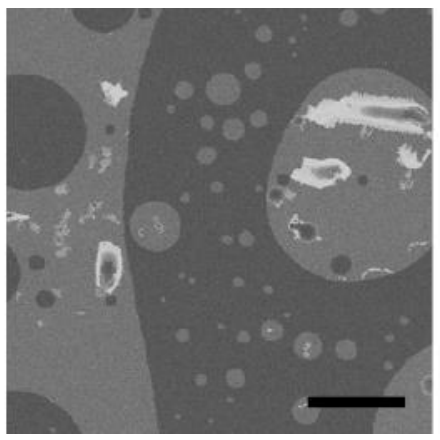

(C)

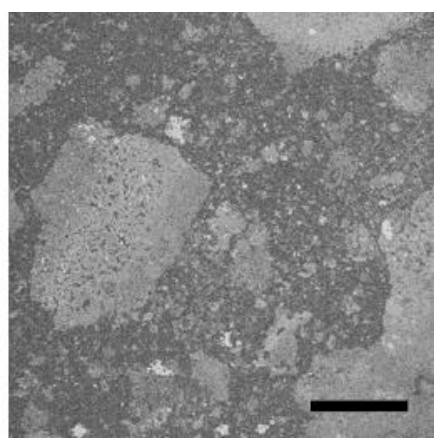

Figure 2. FE-SEM images of liquid IL-based systems: DDT insecticide stored in micro-channels in IL-water mixture (A); different phases of peptide (dialanine) solution in IL-water mixture (B); aggregates in the IL-based reaction mixture for fructose conversion formed in the presence of water (C). Scale bar $-10 \mu \mathrm{m}$. 